\title{
Efficacy and tolerability of vildagliptin as first line treatment in patients with diabetes type 2 in an outpatient setting
}

\author{
M. P. Yavropoulou ${ }^{1,3^{*}}$, M. Pikilidou' ${ }^{2}$, K. Kotsa ${ }^{1}$, A. Michopoulos ${ }^{1}$, E. Papakonstantinou ${ }^{2}$ and J. G. Yovos ${ }^{1}$
}

\begin{abstract}
Background: Inhibitors of dipeptidyl-peptidase IV are recommended as second-line therapy in type 2 diabetes (DT2), but data, as a first-line treatment in everyday clinical practice are scarce. To address this issue we conducted a 12-month, clinical study in an outpatient setting, using vildagliptin as the first-line treatment.

Methods: Ninety-one drug naïve patients with DT2 started with vildagliptin monotherapy (100 mg daily) for 4 months and were scheduled to regular 4-monthly visits for 1 year. Patients received add-on treatment with metformin or metformin and glimepiride according to their glycosylated hemoglobin (HbA1c) at each study-visit.

Results: HbA1c was significantly decreased with vildagliptin monotherapy from $8.16 \% \pm 1.60$ to $7.52 \% \pm 1.60$, $p<0.001$. Only $39 \%$ of the patients achieved the target of HbA1c $\leq 7.0 \%$ at the end of the 4th month. Mean change in $\mathrm{HbA} 1 \mathrm{c}$ was significantly correlated with baseline $\mathrm{HbA1c}$ values $(r=-0.51, p<0.001)$. At the end of the study only $35 \%$ of the patients remained on vildagliptin monotherapy while the rest required add-on treatment with metformin or metformin and sulfonylurea.
\end{abstract}

Conclusions: Vildagliptin is well tolerated either as monotherapy or in combination but the majority of patients require add-on therapy shortly after the beginning of treatment.

Keywords: First line treatment of diabetes type 2, Vildagliptin, DPP-4 inhibitors

\section{Background}

Drugs focusing on the incretin system, such as dipeptidyl peptidase 4 inhibitors (DPP-4i) and glucagon-like peptide -1 (GLP-1) analogues have been recently introduced in the clinical practice for the treatment of diabetes type 2 demonstrating efficacy and a favorable safety profile [1]. Orally administered DPP-4i increase circulating concentrations of endogenous active GLP-1, although to a lesser degree compared to GLP-1 analogues [2] and lower glucose levels by stimulating insulin secretion and inhibiting glucagon secretion.

\footnotetext{
* Correspondence: margia@med.auth.gr

'Division of Endocrinology and Metabolism, 1st Department of Internal Medicine, AHEPA University Hospital, Aristotle University of Thessaloniki, Thessaloniki, Greece

${ }^{3}$ Laboratory of Clinical and Molecular Endocrinology, 1st Department of Internal Medicine, AHEPA University Hospital, 1 S. Kyriakidi street 54636, Thessaloniki, Greece

Full list of author information is available at the end of the article
}

In the recent consensus for the treatment of diabetes [3] DPP-4i, such as vildagliptin and sitagliptin are recommended as second-line therapy in combination with either metformin or sulfonylureas. In patients with diabetes type 2 who do not reach the glycemic targets with metformin alone, DPP-4 inhibitors as add-on treatment can efficiently lower glycosylated hemoglobin (HbA1c), with neutral effects on body weight. Compared with sulfonylureas or thiazolidinediones, DPP-4 inhibitors exert similar hypoglycemic efficacy and in addition are associated with a lower incidence of hypoglycemia or other serious adverse events.

As first line therapy, DPP-4 inhibitors can be an alternative therapeutic option in patients who cannot tolerate metformin because of gastrointestinal adverse events, and several clinical studies have proved their efficacy as monotherapy in drug-naïve patients with diabetes type 2. However, data are scarce regarding DPP-4 inhibitors 
as the first choice for diabetes management in everyday clinical practice.

To address this issue we conducted a clinical study in the outpatient setting using vildagliptin, which is a potent and selective DPP-4i, as the first line treatment in drug -naïve patients with newly diagnosed diabetes type 2 .

\section{Methods}

This was a 12-month clinical study that was conducted in the outpatient setting of the Diabetes center of the Endocrinology Division. All patients with newly diagnosed diabetes type 2 according to WHO criteria that visited the outpatient clinic were initially screened for eligibility. Exclusion criteria were secondary diabetes, prior treatment with oral hypoglycemic agents or insulin, malignancy, acute diabetic complications, myocardial infarction, unstable angina, or coronary artery bypass surgery within the previous 6 months, congestive heart failure, NYHA Class III or IV, liver disease and renal insufficiency $(\mathrm{CrCl}<45 \mathrm{ml} / \mathrm{min})$.

All participants provided written informed consent. The protocol was approved by the ethics committee of AHEPA University Hospital and the study was conducted in accordance with the Declaration of Helsinki.

\section{Study design}

Eligible patients were initially left with lifestyle modification instructions (diet and exercise) for 1-3 months without any other particular treatment. Patients that failed to achieve acceptable glucose levels (ie. FBG $<1120$ and PBG $<200$ ) after this period were finally enrolled and scheduled on regular visits every 4 months for one year. At visit 1 all patients received monotherapy with vildagliptin $(50 \mathrm{mg}$ twice daily) for 4 months. At the end of the fourth month period (visit 2), subjects that were not adequately controlled according to their HbA1c ( $<7.0 \%)$, received add-on therapy with metformin (850 $\mathrm{mg}$ once daily). During the 3rd visit (at the end of the 8th month) patients that did not reach the target of HbA1c $<7.0 \%$ with metformin plus vildagliptin received add-on therapy with a sulfonylurea (Glimepiride 2 or $3 \mathrm{mg}$ twice daily) (Fig. 1). All patients received a BMI adjusted diabetic diet designed by a specialized dietician of the diabetes center in the outpatient clinic that included $50-55 \%$ of carbohydrates, $30 \%$ fat and $15-20 \% \mathrm{f}$ proteins. The calculation of the daily kcals was performed by a relevant application (Bmapp, iOS Developer program, USA) that uses the BMI and the age of the patient and calculates the ideal weight. Adjustments at each visit were based on changes of the body weight of the patient. Patients who in visit 1 had triglycerides levels above $200 \mathrm{mg} /$ $\mathrm{dl}$ and/or total cholesterol $>240 \mathrm{mg} / \mathrm{dl}$ started treatment with atorvastatin $20 \mathrm{mg}$.

According to the treatment applied during the study patients were classified into 3 groups. Group 1: Received vildagliptin for the whole study period (12 months). Group 2. Received vildagliptin for 4 and vildagliptin plus metformin for the remainder 8 months, Group 3: Received vildagliptin for 4 months, vildagliptin plus metformin for 4 months, and vildagliptin plus metformin plus

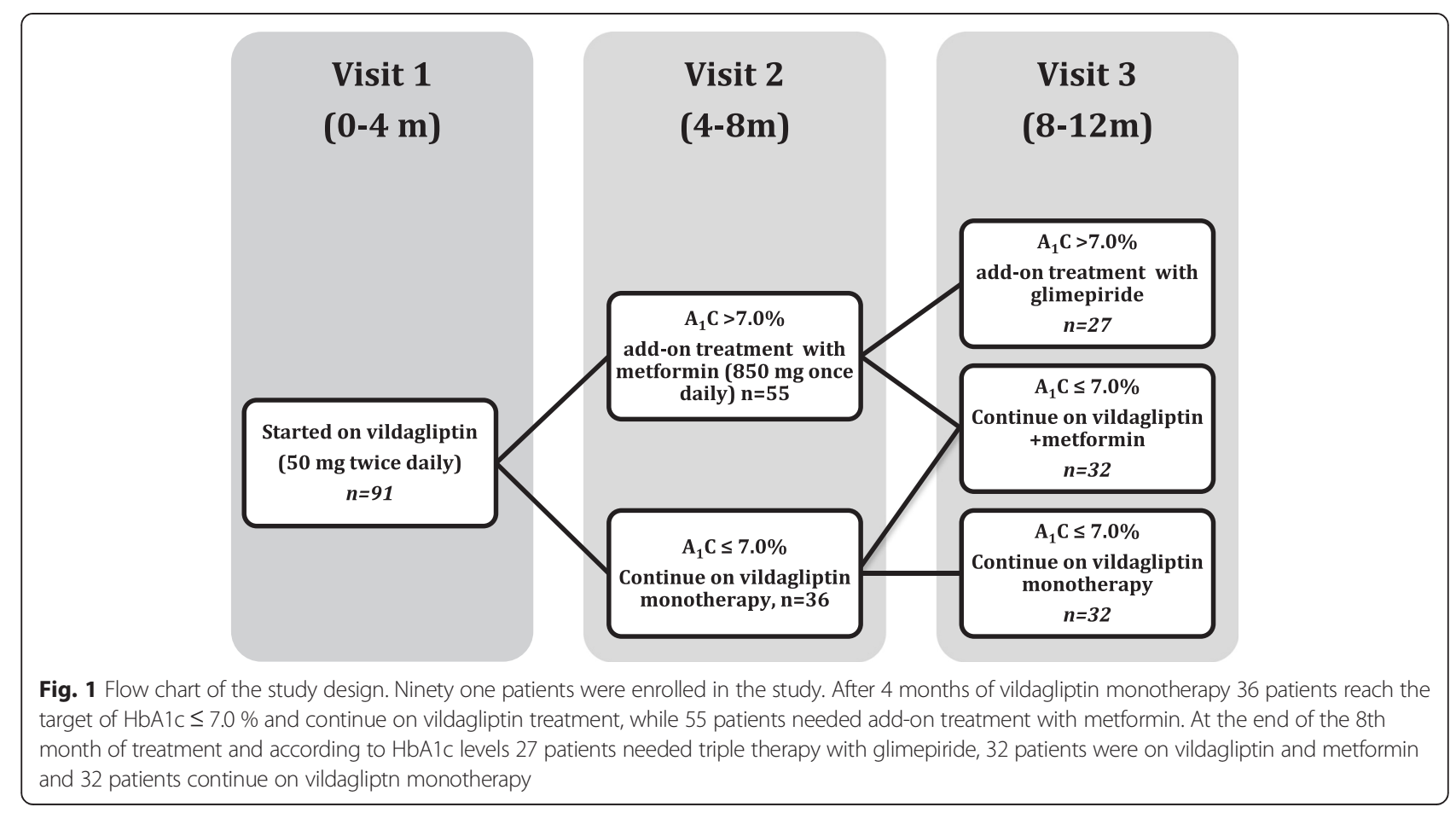


sulfonylurea for the remainder 4 months (Fig. 1). Glycosylated hemoglobin, fasting (FBG) and postprandial blood glucose (PBG), body weight, fasting lipid profiles (triglycerides; TG, total cholesterol; TC, LDL, HDL) standard hematology and biochemistry laboratory assessments and vital signs were measured at each study visit. The PBG at each visit was self-assessed the previous day $2 \mathrm{~h}$ after their regular meal by the glucose-meter provided by the outpatient clinic.

Blood pressure (BP) measurements were performed at each study visit in the morning with the subjects in the seated position following a 5 min quiet resting period. Blood pressure was measured in both arms with a mercury sphygmomanometer using an appropriately sized cuff. Values for systolic BP (SBP) and diastolic BP (DBP) were defined by Korotkoff phase I and IV respectively. All patients were provided with glucose monitoring devices and supplies and instructed on their use during their first visit. Hypoglycemia was defined as selfmonitored blood glucose below $50 \mathrm{mg} / \mathrm{dl}$ with or without symptomatology suggestive of low blood glucose. Adverse events (AEs) were recorded at each study visit and were assessed as to severity using the criteria used by the ICH Guideline for Clinical Safety Data Management (http://www.ema.europa.eu). If an adverse event results in death is life-threatening,- requires inpatient hospitalization or prolongation of existing hospitalization, - results in persistent or significant disability/incapacity, or leads to discontinuation of the treatment is categorized as severe. Classification of mild or moderate adverse events and potential relationship to the anti-diabetic treatment were based on the physicians' opinion taken into consideration the duration of the event and the patient's report.

\section{Assays}

All laboratory assessments were made using standard techniques in the Central Laboratory of AHEPA University hospital. HbA1c was measured with an ion exchange HPLC method.

\section{Statistical analysis}

The primary outcome was change from baseline in HbA1c. Secondary outcomes included fasting and postprandial glucose levels, fasting plasma lipids, and body weight. All values are presented as mean \pm standard deviation (SD), or standard error of the mean (SE) when stated. We used analysis of covariance (ANCOVA) to test whether baseline values of HbA1c were significant in determining the change of HbA1c ( $\triangle \mathrm{HbA1c}$ ), after administration of vildagliptin.

Changes from baseline in primary and secondary endpoints were analyzed using an analysis of variance (ANOVA). Pearson correlation coefficient ( $r$ ) was used to test the relationships between mean changes of glucose parameters after treatment. The trapezoidal rule was used to determine the area under the curve (AUC).

We also used repeated measures analysis to determine whether treatment with vildagliptin was effective over time. Linear regression analysis was performed to create an algorithm that quantifies the net effect of vildagliptin therapy at various values of baseline HbA1c. A $P$ value $<0.05$ was considered statistically significant. Data were analyzed using SPSS 16.0 (SPSS Inc., Chicago, IL, USA).

\section{Results}

Ninety-one patients aged between 39 and 84 years old (mean age 68 years) were finally enrolled in the study. Anthropometric characteristics and baseline laboratory values are summarized in (Table 1 ). Concomitant diseases included arterial hypertension $(n=15)$, thyroid disease $(n=48)$, osteoporosis and other metabolic bone diseases $(n=13)$, hypogonadism $(n=6)$ and hyperlipidemia $(n=2)$. Patients with previously diagnosed hyperlipidemia were on treatment with atorvastatin $20 \mathrm{mg} /$ day for approximately 2 years $(n=2)$ or rosumvastatin $5 \mathrm{mg} /$ day for 3,5 year $(n=1)$ and during laboratory examinations their lipid profile was within normal values. However, at visit one 31 patients had triglycerides $>200 \mathrm{mg} / \mathrm{dl}$ and 25 of them had also total cholesterol $>240 \mathrm{mg} / \mathrm{dl}$. We prescribed atorvastatin $20 \mathrm{mg} / \mathrm{d}$ in these patients. Concomitant medications (including statins) remained unchanged during the study period. Family history of diabetes was positive in $71 \%$ of the patient population. Blood pressure measurements were within normal values during the study period.

Table 1 Baseline and anthropometric characteristics of the study population

\begin{tabular}{ll}
\hline $\mathrm{N}$ & 91 \\
Age (yrs) & $68.4 \pm 11.3$ \\
Men (\%) & $55(60 \%)$ \\
BMl $\left(\mathrm{kg} / \mathrm{cm}^{2}\right)$ & $28 \pm 5.7$ \\
Obese patients (n,\%) & $28,30 \%$ \\
Fasting blood glucose (mmol/l) & $10.3 \pm 2.4$ \\
HbA $\mathrm{C}(\%)$ & $8.16 \pm 1.6$ \\
Postprandial blood glucose (mmol/l) & $11.7 \pm 3.4$ \\
Urea (mmol/l) & $10.67 \pm 2.7$ \\
Creatinine (umol/l) & $79.56 \pm 26.52$ \\
Total Cholesterol (mmol/l) & $5.7 \pm 1$ \\
HDL (mmol/l) & $1.15 \pm 0.3$ \\
LDL (mmol/l) & $3.6 \pm 1.0$ \\
Triglycerides (mmol/l) & $2.17 \pm 1.0$ \\
\hline
\end{tabular}




\section{4 months monotherapy with vildagliptin}

Glycosylated hemoglobin was significantly decreased after 4 months monotherapy with vildagliptin from $8.16 \% \pm 1.60$ at baseline to $7.52 \% \pm 1.60, p<0.001$. The mean change of HbA1c was -0.6 and $39 \%$ of the patients $(n=36)$ achieved the target of HbA1c $\leq 7.0 \%$ at the end of the 4th month. Similarly, significant reductions were observed in FBG, PBG and serum triglycerides level (FBG: $185.5 \pm 43.2 \mathrm{mg} / \mathrm{dl}$ vs. $135 \pm 23.4 \mathrm{mg} / \mathrm{dl}$, $p<0.001$, PBG: $210.8 \pm 61.2 \mathrm{mg} / \mathrm{dl}$ vs. $165.7 \pm 28.8 \mathrm{mg} / \mathrm{dl}$ $p<0.001$, TG: $186 \pm 88.5$ vs. $141.7 \pm 62 \mathrm{mg} / \mathrm{dl}, p<0.001$ ). Total cholesterol levels were also reduced, albeit not significantly, after 4 months of treatment with vildagliptin $(220.4 \pm 38.6 \mathrm{mg} / \mathrm{dl}$ vs. $208.8 \pm 34.8 \mathrm{mg} / \mathrm{dl} p=0.0719)$, while there was no significant change in body weight. Changes in HbA1c were unrelated to changes in FBG and PBG levels.

Patients with baseline HbA1c values $\leq 8.0 \%$ achieved the target of HbA1c $<7.0 \%$ to a significantly greater percentage, compared to patients that had baseline values above $8 \%$ (46\% vs. $25 \%, p<0.001$, respectively). Mean change in HbA1c was significantly and inversely correlated with baseline HbA1c values $(r=-0.51, p<0.001)$, and this relationship remained robust after adjusting for changes in body weight $(\triangle \mathrm{BMI})$, age, gender and baseline FBG and PBG.

A linear regression analysis was used to create a statistical model that quantifies the effect of $\mathrm{HbA1C}$ at baseline on post-treatment $\mathrm{HbA1c}$ and concluded to the following algorithm:

$\triangle \mathrm{HbA} 1 \mathrm{c}=2,40-0,41 \times \mathrm{HbA} \mathrm{c}_{\text {baseline, }}$ which also remained significant $\left(\mathrm{R}^{2}=29,8 \%, p<0,001\right)$, after adjusting for $\triangle \mathrm{BMI}$, age, gender and baseline FBG and PBG.

Mean change in fasting glucose levels $(50.9 \mathrm{mg} / \mathrm{dl})$ was significantly and inversely correlated with baseline values of fasting glucose $(\mathrm{r}=-0.848, p<0.001)$. Mean change in postprandial glucose levels $(37.9 \mathrm{mg} / \mathrm{dl})$ was also significantly correlated with baseline fasting $(\mathrm{r}=-0.269, p=0.029)$ and postprandial glucose levels $(\mathrm{r}=-0.882, p<0.001)$. There was no significant change in body weight.

\section{2 month comparative study with vildagliptin monotherapy versus combination therapy}

During visits 2 and 3 patients either remained on vildagliptin monotherapy $(n=32)$ or received add-on treatment with metformin $(n=32)$ or received add-on treatment with metformin and glimepiride $(n=27)$ according to whether the HbA1c target of below $7.0 \%$ was achieved (Fig. 1).

Repeated measurements analysis for group 1 that received vildagliptin monotherapy during the whole study period, confirmed that there was no difference on the effect of vildagliptin overtime and that was true for both males and females. There was no significant difference between baseline values of fasting and postprandial glucose and fasting lipids between the 3 groups, except for HbA1c, as expected by study design (Table 2). Similarly there were no significant between-groups differences in mean changes in FBG and PBG (Table 3). However, when we used AUC for the assessment of fluctuation of HbA1c at the study intervals, AUC ${ }_{\text {HBA1C } 1-12}$ was significantly higher in group 3 compared to groups 2 and 1 $(6.27 \pm 0.66 \%$ vs. $5.54 \pm 0.43 \%$ vs. $4.7 \pm 0.44, p<0.001$, respectively). This was not the case when assessing AUC

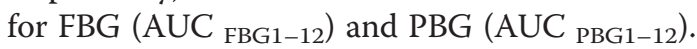

At the end of study period $90 \%$ of the patients in the vildagliptin monotherapy group, $56 \%$ in the vildagliptin plus metformin group and $44 \%$ of the vildagliptin plus metformin plus sulfonylurea group achieved the target of $\mathrm{HbA} 1 \mathrm{c}<7.0 \%$.

Table 2 Baseline values of diabetic patients treated with vildagliptin monotherapy versus ccombination treatment

\begin{tabular}{|c|c|c|c|c|}
\hline Baseline values & Group 1 (Vildagliptin) & Group 2 (Vildagliptin +metformin) & Group 3 (Vildagliptin+metformin +glimepiride) & $P$ \\
\hline $\mathrm{N}$ & 32 & 32 & 27 & - \\
\hline Age (yrs) & $59.7 \pm 10.2$ & $63.1 \pm 12.3$ & $65 \pm 10.5$ & 0.23 \\
\hline Men (\%) & $20(62 \%)$ & $22(68 \%)$ & $13(48 \%)$ & - \\
\hline BMI $\left(\mathrm{kg} / \mathrm{cm}^{2}\right)$ & $28 \pm 5$ & $27 \pm 4.6$ & $29.6 \pm 7.5$ & 0.34 \\
\hline Fasting blood glucose (mmol/l) & $10.5 \pm 2.9$ & $10.4 \pm 2.1$ & $9.7 \pm 1.9$ & 0.64 \\
\hline $\mathrm{HbA1c}(\%)$ & $7.4 \pm 1.3$ & $8.1 \pm 1.6$ & $9 \pm 1.3$ & $<0.001$ \\
\hline Postprandial glucose (mmol/l) & $11.2 \pm 3.6$ & $11.65 \pm 2.9$ & $12 \pm 3.7$ & 0.23 \\
\hline Total Cholesterol (mmol/l) & $6.1 \pm 0.9$ & $5.6 \pm 1.2$ & $5.8 \pm 0.8$ & 0.46 \\
\hline $\mathrm{HDL}(\mathrm{mmol} / \mathrm{l})$ & $1.1 \pm 0.2$ & $1.2 \pm 0.3$ & $1.1 \pm 0.3$ & 0.30 \\
\hline LDL (mmol/l) & $3.7 \pm 0.9$ & $3.3 \pm 1.1$ & $3.6 \pm 0.8$ & 0.41 \\
\hline Triglycerides (mmol/l) & $1.9 \pm 0.6$ & $2.3 \pm 1.3$ & $2.3 \pm 0.9$ & 0.37 \\
\hline Systolic Blood pressure (mmHg) & $120.9 \pm 8.3$ & $118.9 \pm 7.2$ & $121 \pm 8.4$ & 0.33 \\
\hline Diastolic Blood pressure (mmHg) & $80.6 \pm 7.3$ & $79.9 \pm 6.8$ & $89.6 \pm 7.3$ & 0.47 \\
\hline
\end{tabular}


Table 3 Mean changes in glucose and lipids before and after treatment for 12 months

\begin{tabular}{|c|c|c|c|}
\hline Mean change of tested parameters & Group 1 & Group 2 & Group 3 \\
\hline Mean \pm SE & Vildagliptin monotherapy & Vildagliptin +Metformin & Vildagliptin+ Metformin +Glimepiride \\
\hline Mean change in $\mathrm{HbA} 1 \mathrm{C}(\%)$ & $0.9 \pm 0.22 \% p<0.001$ & $1.2 \pm 0.27 \% p<0.001$ & $1.4 \pm 0.25 \% p<0.001$ \\
\hline Mean change FBG (mg/dl) & $68.57 \pm 8.5 \mathrm{mg} / \mathrm{dl} p<0.001$ & $55.17 \pm 9.6 \mathrm{mg} / \mathrm{dl} p<0.001$ & $101 \pm 14 \mathrm{mg} / \mathrm{dl} p<0.001$ \\
\hline Mean change PBG (mg/dl) & $-37.24 \pm 10 \mathrm{mg} / \mathrm{dl} p<0.005$ & $56.4 \pm 13 \mathrm{mg} / \mathrm{dl} p<0.001$ & $120.2 \pm 24.2 p<0.001$ \\
\hline Mean change triglycerides (mg/dl) & $24.7 \pm 16 \mathrm{mg} / \mathrm{dl} p<0.001$ & $52 \pm 21 \mathrm{mg} / \mathrm{dl} p=0.01$ & $71 \pm 33 \mathrm{mg} / \mathrm{dl} p=0.27$ \\
\hline Mean change total cholesterol (mg/dl) & $12 \pm 2 \mathrm{mg} / \mathrm{dl} p=0.32$ & $13 \pm 10 \mathrm{mg} / \mathrm{dl} p=0.21$ & $17 \pm 9 \mathrm{mg} / \mathrm{dl} p=0.06$ \\
\hline Mean change $\mathrm{HDL}(\mathrm{mg} / \mathrm{dl})^{a}$ & $6.47 \pm 4 \mathrm{mg} / \mathrm{dl} p=0.02$ & $4 \pm 3 \mathrm{mg} / \mathrm{dl} p=36$ & $10 \pm 5 \mathrm{mg} / \mathrm{dl} p=0.26$ \\
\hline Mean change LDL (mg/dl) & $20.4 \pm 6 \mathrm{mg} / \mathrm{dl} p=0.77$ & $41 \pm 18 \mathrm{mg} / \mathrm{dl} p=0.93$ & $50 \pm 13 \mathrm{mg} / \mathrm{dl} p=0.08$ \\
\hline Mean change BW (kg) & $0.20 .2 \pm 0.03 p=0.32$ & $1.2 \pm 0.21 .2 p=0.4$ & $1.3 \pm 0.6 \mathrm{~kg} 1.3 p=0.48$ \\
\hline
\end{tabular}

a $\mathrm{HDL}$ level was increased after treatment

\section{Mean changes of the parameters tested at the end of the study}

Mean changes in glucose levels, HbA1c, lipids and weight are shown in Table 3 . The mean change \pm SE in HbA1c from baseline to end point was larger in group 3 $(-1.4 \pm 0.25 \%)$, compared to group $2(-1.2 \pm 0.27 \%)$ and group $1(-0.9 \pm 0.22 \%)$, albeit not significantly (Table 3$)$.

There was a significant reduction in FBG and PBG at the end of the study in all groups. Triglycerides and total cholesterol levels were significantly reduced in group 1 and group 2 while significant changes were seen in HDL and LDL levels in groups 1 and 3, respectively.

\section{Adverse events}

No confirmed symptomatic or asymptomatic hypoglycemia was reported during the study period. Most of the AEs that were reported were mild or moderate in severity, did not lead to discontinuation of the anti-diabetic treatment and included nausea $(n=12)$ and gastrointestinal discomfort $(n=5)$. One patient in the vildagliptin monotherapy group reported an oedema in the lower extremities, which was resolved in the first month of treatment.

In addition no adverse reactions attributed to drug- interactions were reported during the study period. No major changes from baseline to endpoint were observed for any of the routinely assessed hematological or biochemical parameters.

\section{Discussion}

The efficacy and safety of vildagliptin as monotherapy have been widely confirmed in a large body of clinical studies in various populations with type 2 diabetes [4-8]. In our study and in agreement with previous data, vildagliptin monotherapy (100 mg daily) produced a clinically significant decrease in HbA1c as a first-line treatment in drugnaive patients with type 2 diabetes. However, the reduction of HbA1c observed was lower in the older age patients probably due to the longer duration of diabetes in this group and was also lower compared to randomized clinical trials, probably due to the nature of our study design that do not allow us a tight control on the patient's diet and compliance to study drugs, which were both selfreported.

Moreover we have shown a favorable lipid profile in all three groups of treatment with significant reductions in serum triglycerides and LDL cholesterol and significant increases in HDL in combination with atorvastatin.

A small number of patients, achieved the target of $7.0 \%$ in the first 4 months of vildagliptine monotherapy (36\%) but most of them (approximately $90 \%$ ) sustain HbA1c $<7.0 \%$ at least for 1 year after the first diagnosis of their diabetes, in line with previous results demonstrating a sustained effect of vildagliptin over time [8]. At the end of study period $90 \%$ of the patients in the vildagliptin monotherapy group, $56 \%$ in the vildagliptin plus metformin group and $44 \%$ of the vildagliptin plus metformin plus sulfonylurea group achieved the target of $\mathrm{HbA} 1 \mathrm{c}<7.0 \%$. Based on the algorithm that was developed by data from our patients we found that the main determinant of vildagliptin efficacy as monotherapy in drug-naïve patients was, baseline HbA1c as it has also been shown for vildagliptin plus metformin [9]. In a recent study by Takeshita et al., when vildagliptin was compared to liraglutide as a second line treatment after sitagliptin baseline levels of docosahexanoic acid but not eicosapentanoic acid demonstrated a significant predictive value for vildagliptinmediated improvement in glycemic control independent of its effects on insulin secretion and insulin sensitivity [10]. Detailed lipid parameters were not measured in our study. In addition, our patients were drug naïve when vildagliptine was initiated and thus our results are not directly comparable with the study by Takeshita et al. [10].

Vildagliptin was well tolerated either as monotherapy or in combination with metformin or metformin and glimepiride, and no episodes of hypoglycemia were observed. 
Currently, most type 2 diabetes patients begin and continue with the gold standard treatment of metformin [11]. When metformin is not tolerated due to gastrointestinal side effects, such as diarrhea and nausea [12] or is contraindicated, as in congestive heart failure and renal disease, alternative first line treatments include sulfonylureas, thiazolidinediones and DPP-4i. Compared to the first two drug categories DPP-4i are equally efficacious in lowering $\mathrm{HbA} 1 \mathrm{c}$ and have a favorable safety profile with low risk of hypoglycemia due to the incretin-based mechanism of action and neutral effect on body weight.

In studies comparing vildagliptin to metformin vildagliptin has proven non-inferiority and was better tolerated with fewer gastrointestinal adverse events [13-15].

Clinically relevant efficacy of vildagliptin was also seen compared to rosiglitazone [16-18]. Both drugs decreased HbA1c to a similar extent at short term with vildagliptin showing some weight benefit [16]. Extension of this study [16] over 2-years in drug-naïve diabetic patients also demonstrated statistically significant and sustained HbA1c reductions for both drugs with rosiglitazone showing greater durability in lowering HbA1c at the expense of significant weight gain and less favorable plasma lipid profile compared with vildagliptin [17].

Compared to gliclazide, vildagliptin produced similar HbA1c reductions over 2-years monotherapy but noninferiority was not established [19] despite the benefits of vildagliptin in weight and hypoglycemia. In patients with low baseline values of HbA1c vildagliptin and glimepiride as add on treatment to metformin demonstrated similar efficacy in reducing $\mathrm{HbA1c}$ with markedly reduced hypoglycemia risk and no weight gain in the vildagliptin plus metformin treated group [20,21].

Overall, monotherapy with vildagliptin or vildagliptin plus metformin controlled adequately HbA1c levels in $73 \%$ of drug-naïve patients with newly diagnosed diabetes after 12 months of treatment in line with previous results $[4,9,22-24]$. Patients that needed add-on treatment with sulfonylurea were those with more pronounced postprandial hyperglycemia and mean baseline values of HbA1c around $9 \%$. We did not find significant differences between treatment groups from baseline to end point in the glucose parameters tested, probably due to the small sample size in each group.

\section{Conclusions}

In summary we have shown that monotherapy of vildagliptin as first line treatment or in combination with metformin in the outpatient setting is an effective approach in a considerable number of patients albeit with an increased cost relative to more conventional alternatives. Additional benefits such as low risk of hypoglycemia, neutral effect on body weight and favorable lipid profile can be particularly appreciable in selective patients with low baseline
HbA1c levels or in the elderly population, as has been recently shown [25].

\section{Competing interests}

The authors declare that they have no competing interest.

\section{Authors' contributions}

MY: collecting data, analyzing data, writing the paper, MP: collecting data, analyzing data, writing the paper, KK: revising the paper, AM: statistical analysis, EP: collecting data, JY: revising the paper. All authors read and approved the final manuscript.

\section{Author details}

${ }^{1}$ Division of Endocrinology and Metabolism, 1st Department of Internal Medicine, AHEPA University Hospital, Aristotle University of Thessaloniki, Thessaloniki, Greece. ${ }^{2} 1$ st Department of Internal Medicine, AHEPA University Hospital, Aristotle University of Thessaloniki, Thessaloniki, Greece. ${ }^{3}$ Laboratory of Clinical and Molecular Endocrinology, 1st Department of Internal Medicine, AHEPA University Hospital, 1 S. Kyriakidi street 54636, Thessaloniki, Greece.

Received: 27 April 2015 Accepted: 12 August 2015

Published online: 22 August 2015

\section{References}

1. Drucker DJ, Nauck MA. The incretin system: glucagon-like peptide-1 receptor agonists and dipeptidyl peptidase-4 inhibitors in type 2 diabetes. Lancet. 2006;368(9548):1696-705. PubMed.

2. Ahren $B$, Simonsson $E$, Larsson $H$, Landin-Olsson M, Torgeirsson $H$, Jansson PA, et al. Inhibition of dipeptidyl peptidase IV improves metabolic control over a 4-week study period in type 2 diabetes. Diabetes Care. 2002;25(5):869-75. PubMed.

3. Inzucchi SE, Bergenstal RM, Buse JB, Diamant M, Ferrannini E, Nauck M, et al. Management of hyperglycemia in type 2 diabetes, 2015: a patient-centered approach: update to a position statement of the American Diabetes Association and the European Association for the Study of Diabetes. Diabetes Care. 2015;38(1):140-9. PubMed.

4. Dejager S, Razac S, Foley JE, Schweizer A. Vildagliptin in drug-naive patients with type 2 diabetes: a 24-week, double-blind, randomized, placebo-controlled, multiple-dose study. Horm Metab Res. 2007;39(3):218-23. PubMed.

5. Pi-Sunyer FX, Schweizer A, Mills D, Dejager S. Efficacy and tolerability of vildagliptin monotherapy in drug-naive patients with type 2 diabetes. Diabetes Res Clin Pract. 2007;76(1):132-8. PubMed.

6. Foley JE, Bunck MC, Moller-Goede DL, Poelma M, Nijpels G, Eekhoff EM, et al. Beta cell function following 1 year vildagliptin or placebo treatment and after 12 week washout in drug-naive patients with type 2 diabetes and mild hyperglycaemia: a randomised controlled trial. Diabetologia. 2011;54(8):1985-91. PubMed Pubmed Central PMCID: 3131517.

7. Scherbaum WA, Schweizer A, Mari A, Nilsson PM, Lalanne G, Jauffret S, et al. Efficacy and tolerability of vildagliptin in drug-naive patients with type 2 diabetes and mild hyperglycaemia*. Diabetes Obes Metab. 2008;10(8):675-82. PubMed.

8. Scherbaum WA, Schweizer A, Mari A, Nilsson PM, Lalanne G, Wang Y, et al. Evidence that vildagliptin attenuates deterioration of glycaemic control during 2-year treatment of patients with type 2 diabetes and mild hyperglycaemia. Diabetes Obes Metab. 2008;10(11):1114-24. PubMed.

9. Bosi E, Dotta F, Jia Y, Goodman M. Vildagliptin plus metformin combination therapy provides superior glycaemic control to individual monotherapy in treatment-naive patients with type 2 diabetes mellitus. Diabetes Obes Metab. 2009;11(5):506-15. PubMed.

10. Takeshita Y, Takamura T, Kita Y, Otoda T, Kato K, Wakakuri H, et al. Vildagliptin vs liraglutide as a second-line therapy switched from sitagliptin-based regimens in patients with type 2 diabetes: a randomized, parallel-group study. J Diabetes Investig. 2015;6(2):192-200. PubMed Pubmed Central PMCID: 4364854.

11. Halimi S. Metformin: 50 years old, fit as a fiddle, and indispensable for its pivotal role in type 2 diabetes management. Diabetes Metab. 2006;32(6):555-6. PubMed.

12. Bouchoucha M, Uzzan B, Cohen R. Metformin and digestive disorders. Diabetes Metab. 2011;37(2):90-6. PubMed.

13. Goke B, Hershon K, Kerr D, Calle Pascual A, Schweizer A, Foley J, et al. Efficacy and safety of vildagliptin monotherapy during 2-year treatment of 
drug-naive patients with type 2 diabetes: comparison with metformin Horm Metab Res. 2008;40(12):892-5. PubMed.

14. Schweizer A, Couturier A, Foley JE, Dejager S. Comparison between vildagliptin and metformin to sustain reductions in $\mathrm{HbA}(1 \mathrm{c})$ over 1 year in drug-naive patients with type 2 diabetes. Diabet Med. 2007;24(9):955-61. PubMed.

15. Schweizer A, Dejager S, Bosi E. Comparison of vildagliptin and metformin monotherapy in elderly patients with type 2 diabetes: a 24-week, double-blind, randomized trial. Diabetes Obes Metab. 2009;11(8):804-12. PubMed.

16. Rosenstock J, Baron MA, Dejager S, Mills D, Schweizer A. Comparison of vildagliptin and rosiglitazone monotherapy in patients with type 2 diabetes: a 24-week, double-blind, randomized trial. Diabetes Care. 2007;30(2):217-23. PubMed.

17. Rosenstock J, Niggli M, Maldonado-Lutomirsky M. Long-term 2-year safety and efficacy of vildagliptin compared with rosiglitazone in drug-naive patients with type 2 diabetes mellitus. Diabetes Obes Metab. 2009;11(6):571-8. PubMed.

18. Montori VM. Vildagliptin was noninferior to rosiglitazone for glycemic control in type 2 diabetes but caused less weight gain. ACP J Club. 2007;146(3):74. PubMed.

19. Foley JE, Sreenan S. Efficacy and safety comparison between the DPP-4 inhibitor vildagliptin and the sulfonylurea gliclazide after two years of monotherapy in drug-naive patients with type 2 diabetes. Horm Metab Res. 2009;41(12):905-9. PubMed

20. Matthews DR, Dejager S, Ahren B, Fonseca V, Ferrannini E, Couturier A, et al. Vildagliptin add-on to metformin produces similar efficacy and reduced hypoglycaemic risk compared with glimepiride, with no weight gain: results from a 2-year study. Diabetes Obes Metab. 2010;12(9):780-9. PubMed.

21. Ferrannini E, Fonseca V, Zinman B, Matthews D, Ahren B, Byiers S, et al. Fifty-two-week efficacy and safety of vildagliptin vs. glimepiride in patients with type 2 diabetes mellitus inadequately controlled on metformin monotherapy. Diabetes Obes Metab. 2009;11(2):157-66. PubMed.

22. Ahren B. Novel combination treatment of type 2 diabetes DPP-4 inhibition + metformin. Vasc Health Risk Manag. 2008;4(2):383-94. PubMed Pubmed Central PMCID: 2496991.

23. Pratley RE, Fleck P, Wilson C. Efficacy and safety of initial combination therapy with alogliptin plus metformin versus either as monotherapy in drug-naive patients with type 2 diabetes: a randomized, double-blind, 6-month study. Diabetes Obes Metab. 2014;16(7):613-21. PubMed.

24. Wu D, Li L, Liu C. Efficacy and safety of dipeptidyl peptidase-4 inhibitors and metformin as initial combination therapy and as monotherapy in patients with type 2 diabetes mellitus: a meta-analysis. Diabetes Obes Metab. 2014;16(1):30-7. PubMed.

25. Strain WD, Lukashevich V, Kothny W, Hoellinger MJ, Paldanius PM. Individualised treatment targets for elderly patients with type 2 diabetes using vildagliptin add-on or lone therapy (INTERVAL): a 24 week, randomised, double-blind, placebo-controlled study. Lancet. 2013;382(9890):409-16. PubMed.

\section{Submit your next manuscript to BioMed Central and take full advantage of:}

- Convenient online submission

- Thorough peer review

- No space constraints or color figure charges

- Immediate publication on acceptance

- Inclusion in PubMed, CAS, Scopus and Google Scholar

- Research which is freely available for redistribution 